\title{
Screening of health workers exposed to SARS-CoV-2 in a university hospital in the south of France
}

\author{
Ludovic Noel $^{1}$ - Elise Marion ${ }^{1} \cdot$ Rafika Boufercha $^{1} \cdot$ Françoise Martin ${ }^{1} \cdot$ Christine Zandotti $^{2} \cdot$ Remi Charrel $^{2}$. \\ Mouloud Bouhadfane ${ }^{1} \cdot$ Marie Pascale Lehucher-Michel ${ }^{1,3} \cdot$ Antoine Villa $^{1}[$
}

Received: 10 March 2021 / Accepted: 16 August 2021 / Published online: 15 October 2021

(c) The Author(s), under exclusive licence to Springer-Verlag GmbH Germany, part of Springer Nature 2021

\begin{abstract}
Introduction SARS-CoV-2, which causes COVID-19, is a virus that has caused a global pandemic. Health workers (HWs) are major players in the fight against this infection and are occupationally exposed to the virus in the course of their work. In this context, this study presents surveillance data on 1714 workers in a hospital center in the south of France for the period from March 17 to April 20, 2020.

Materials and methods Symptomatic HWs, contact cases and those with high anxiety were tested. Diagnosis of COVID-19 was performed by RT-PCR after nasopharyngeal sampling.

Results During this period, $30.4 \%$ of hospital staff received 3028 nasal swabs. Of these, $8.0 \%$ were infected with SARSCoV-2. Among the SARS-CoV-2 positive HWs, $24.3 \%$ were asymptomatic. Among COVID unit and non COVID unit, the positive HWs for SARS-CoV-2 were, respectively, 5.8\% and $8.2 \%(p=0.2)$. HWs over 50 years of age were less likely to be positive for SARS-CoV-2 $(3.8 \%)$ than other younger HWs $(9.1 \%)(p<0.001)$. No serious cases of COVID-19 were reported in our population during this period.

Discussion Our study suggests that HWs who tested positive for COVID-19 are often asymptomatic. Therefore, PPE is pivotal to prevent HWs to patients and HWs to HWs transmission during workshifts. Contact tracing and screening is essential to limit the spread of the virus within the hospital. On the other hand, HWs working in COVID-19 units are not more often infected probably because they have a higher risk awareness than other HWs.
\end{abstract}

Keywords COVID-19 $\cdot$ SARS-CoV-2 $\cdot$ Nurse $\cdot$ Healthcare worker $\cdot$ Hospital

\section{Introduction}

SARS-CoV-2 is a virus responsible for a pandemic (COVID19) that began on December 31, 2019 in Wuhan, China. Within weeks, the virus spread throughout China and within a month to several other countries, including Italy, the United States and Germany (Fan et al. 2020).

Antoine Villa

antoine.villa@ap-hm.fr

1 Service de Médecine et Santé au Travail, Hôpital de la Timone, APHM, 264, Rue Saint Pierre, 13005 Marseille, France

2 Laboratoire de Virologie, IHU Méditerranée Infection, CLIN, CHU Timone, UMR 190-Emergence des Pathologies Virales, Marseille, France

3 Aix-Marseille Univ, SPMC EA 3279, Marseille, France
Symptoms of COVID-19 can range from a mild flu-like illness to severe acute respiratory distress syndrome (Tostmann et al. 2020; Tu et al. 2020). Approximately 3-29\% of these patients are admitted to an intensive care unit. The overall mortality rate is approximately 5.4\% (Tu et al. 2020).

Health workers (HWs) are key players in the fight against this pandemic (Lombardi et al. 2020). Their occupational exposure to the virus occurs when they care for infected patients, but also when they come into contact with other HWs who carry the virus. In this context, the implementation of collective and individual prevention measures is essential to limit the risk of contamination (Çelebi et al. 2020). To verify the effectiveness of these measures, a screening strategy for symptomatic subjects and contact subjects is necessary.

In this context, the aim of our study was to identify the number of positive HWs among the staff of a University 
Hospital Center in the south of France who had been screened by RT-PCR for SARS-CoV-2.

\section{Material and method}

This descriptive cross-sectional study was performed on HWs in a hospital center in the south of France.

\section{Population studied}

All HWs who consulted an occupational physician from March 17 to April 20, 2020 for an RT-PCR test were included. The criteria for carrying out test were as follows: (1) symptomatic HWs (2) HWs, who were contact cases, were tested 4 days and 7 days after contact if they were asymptomatic or as soon as symptoms appeared; (3) HWs presenting with great anxiety about the possibility of infection and seeking reassurance. A HW was considered symptomatic if he presented otolaryngological or pulmonary symptoms or general signs such as fever, aches, arthralgias, myalgias.

A sample was collected by a nurse of Occupational Health and Medicine Department and sent to the virology department for RT-PCR testing. Results were obtained within $24 \mathrm{~h}$. When a HW had a positive RT-PCR test, a specialized consultation by an infectious disease specialist was offered.

\section{Nature of the tests performed}

Viral RNA was extracted from $200 \mu \mathrm{L}$ of nasopharyngeal swab fluid, using the EZ1 Virus Mini Kit v2.0 (Qiagen ${ }^{\circledR}$, Courtaboeuf, France). Then, SARS-CoV-2 RNA was assessed by a real-time reverse transcription (RT)-PCR system targeting the envelope protein (E)-encoding gene with the LightCycler Multiplex RNA Virus Master kit on a LightCycler 480 instrument (Roche Diagnostics, Mannheim, Germany). Estimated detection threshold was 200 copies $/ \mathrm{mL}$. An internal control phage was used to ensure RNA extraction and PCR accuracies (Lagier et al. 2020). A cycle threshold (CT) value $<35$ is defined as positive. The RT-PCR tests used in this study had a very high specificity (>99\%).

\section{Statistical analysis}

Statistical analysis was performed using SPSS 20.0 software. A Chi-square test and a Student test were used to data analysis.

\section{Results}

The hospital had a workforce of 5704 HWs of whom 1714 $(30.0 \% ; 1714 / 5704)$ were sampled and testing within the study period. Of the 3028 samples collected from the 1714 HWs tested, $8.0 \%$ (137/1714) were positive. Of the HWs tested: 893 had one sample, 634 had 2 samples, 75 had 3 samples and 117 had 4 samples or more.

The peak period of activity was from March 23 to March 30, 2020. During this period the median number of samples was 193 samples per day with a minimum of 44 and a maximum of 240 samples per day. The time course of the number of samples and positive tests between March 17 and April 20 is shown in Fig. 1.

The comparison of the evolution of the incidence of COVID in France and the number of tests performed by hospital staff is shown in Fig. 2.

The population tested consisted of $72.0 \%$ females (1234/1714). Of the 137 positive HWs, 67.1\% (92/137) females were positive. There was no significant difference between the gender distribution in the population tested and the positive HWs $(p=0.23)$.

Among the men tested, 9.4\% (45/480) were positive for SARS-CoV2. Among the women tested, 7.5\% (92/1234) were positive for SARS-CoV-2. There was no significant difference between males and females RT-PCR positive $(p=0.2)$.

The distribution of SARS-CoV-2 positive HWs by age is shown in Fig. 3.

The HWs tested were younger (mediane 37 years; min-max 20-72 years) than the the entire hospital population (mediane 39 years; $\min -\max 20-72$ years) $(p<0.001)$. In the population tested by RT-PCR, the positive HWs were younger (mediane 32 years; min-max 22-62 years) than the negative HWs (mediane 37 years; min-max 21-72 years) $(p<0.001)$.

HWs over 50 years of age were less likely to be positive for SARS-CoV-2 $(3.8 \%$; 14/373) than younger HWs $(9.2 \%$; 123/1341) (Fig. 2) $(p<0.001)$.

Among the SARS-CoV2 positive HWs, nurses, residents/physicians and assistant-nurses were the most affected occupational categories with $29.1 \%$ (40/137) and $21.2 \%$ (29/137) and 10.9\% (15/137) respectively. Among physicians, the majority were resident $(14.6 \%$; 20/137).

Among COVID unit and non COVID unit, the positive HWs for SARS-CoV-2 was respectively 5.8\% (13/223) and $8.2 \%(124 / 1512)(p=0.2)$. Among SARS-CoV-2 positive HWs working off COVID unit, $6.4 \%(12 / 188)$ were assigned to pediatric units and $8.1 \%(125 / 1547)$ were assigned to adult or laboratory units $(p=0.4)$. Of the HW from adult units, $6.4 \%$ (8/124) were from Internal 
300

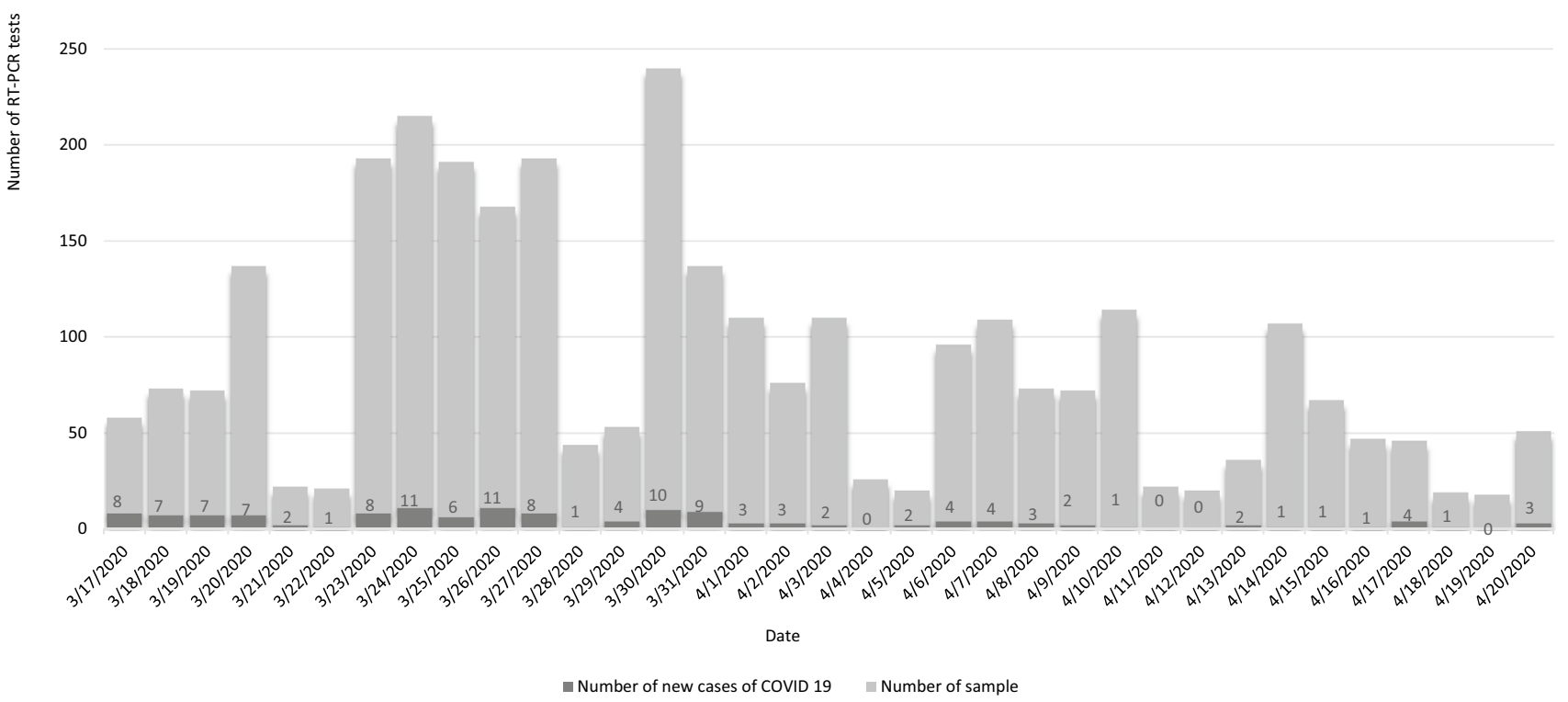

Fig. 1 Chronological evolution of the number of RT-PCR tests performed between 17/03/2020 and 20/04/2020. The period of greatest activity was from 24 March to 30 March, during which the number of new covid 19 cases did not increase in proportion to the number of tests performed

Medicine, 5.6\% (7/124) from Neurology, 5.6\% (7/124) from Pharmacy and $5.6 \%$ from Emergency Medical Service.

Among HWs with at least one positive SARS-CoV-2 sample, $24.3 \%$ (25/103) were asymptomatic at the time of the positive test.

RT-PCR tests $(35.9 \%$; 952/2649) were performed in symptomatic HWs; $8.1 \%$ (77/952) of these tests were positive. RT-PCR tests $(38.1 \% ; 1010 / 2649)$ were performed in HWs, without symptom, who had risky contact, i.e. contact with a COVID-19 positive person, at less than $1 \mathrm{~m}$ distance, for more than $15 \mathrm{~min}$, without a mask; $2.3 \%$ (23/1010) of these tests were positive. RT-PCR test performed in symptomatic HWs were more likely to be positive than RT-PCR test performed in asymptomatic HWs with risky contact $(p<0.001)$. RT-PCR tests $(25.9 \%$; $687 / 2649)$ were also performed in asymptomatic anxious HWs; $0.3 \%$ (2/687) of these tests were positive. RT-PCR test performed in asymptomatic HWs with risky contacts were more likely to be positive than RT-PCR test performed in asymptomatic anxious HWs $(p<0.001)$.

Among the positive RT-PCR tests, there was no significant difference $(p=0.36)$ between the CT values of symptomatic HWs (mean $25.0 \pm 5.4$ ) and the CT values of asymptomatic HWs (mean $25.5 \pm 5.5$ ).

During this period there was no reports of severe SARS-CoV-2 infection among hospital staff.

\section{Discussion}

Limited French data are available on the contamination of HWs exposed to SARS-CoV2 (Greffe et al. 2020; Krastinova et al. 2020; Gagneux-Brunon et al. 2020).

Few HWs at the hospital contracted SARS-CoV2. This finding is equivalent to those of seven studies that tested both symptomatic and asymptomatic HWs in contact with positive cases (Tostmann et al. 2020; Lombardi et al. 2020; Çelebi et al. 2020; Reusken et al. 2020; Rivett et al. 2020; Barrett et al. 2020; Porru et al. 2020). But this result is lower than that of three studies (Hunter et al. 2020; Keeley et al. 2020; Leeds et al. 2020) that tested only symptomatic cases; they found $14 \%, 18 \%$ and $43 \%$ positive HWs respectively.

More than $20 \%$ of positive healthcare workers were asymptomatic. This finding was also reported in two studies (Rivett et al. 2020; Lombardi et al. 2020) since respectively $57 \%$ and $20.1 \%$ of the positive HWs in their series were asymptomatic. These data highlight the importance of systematic screening of even asymptomatic contact cases and the use of Personal Protective Equipment (PPE) to avoid transmission.

Contact tracing and screening is essential to limit the spread of the virus within the hospital. From an epidemiological point of view, the simple anxiety of HWs should 


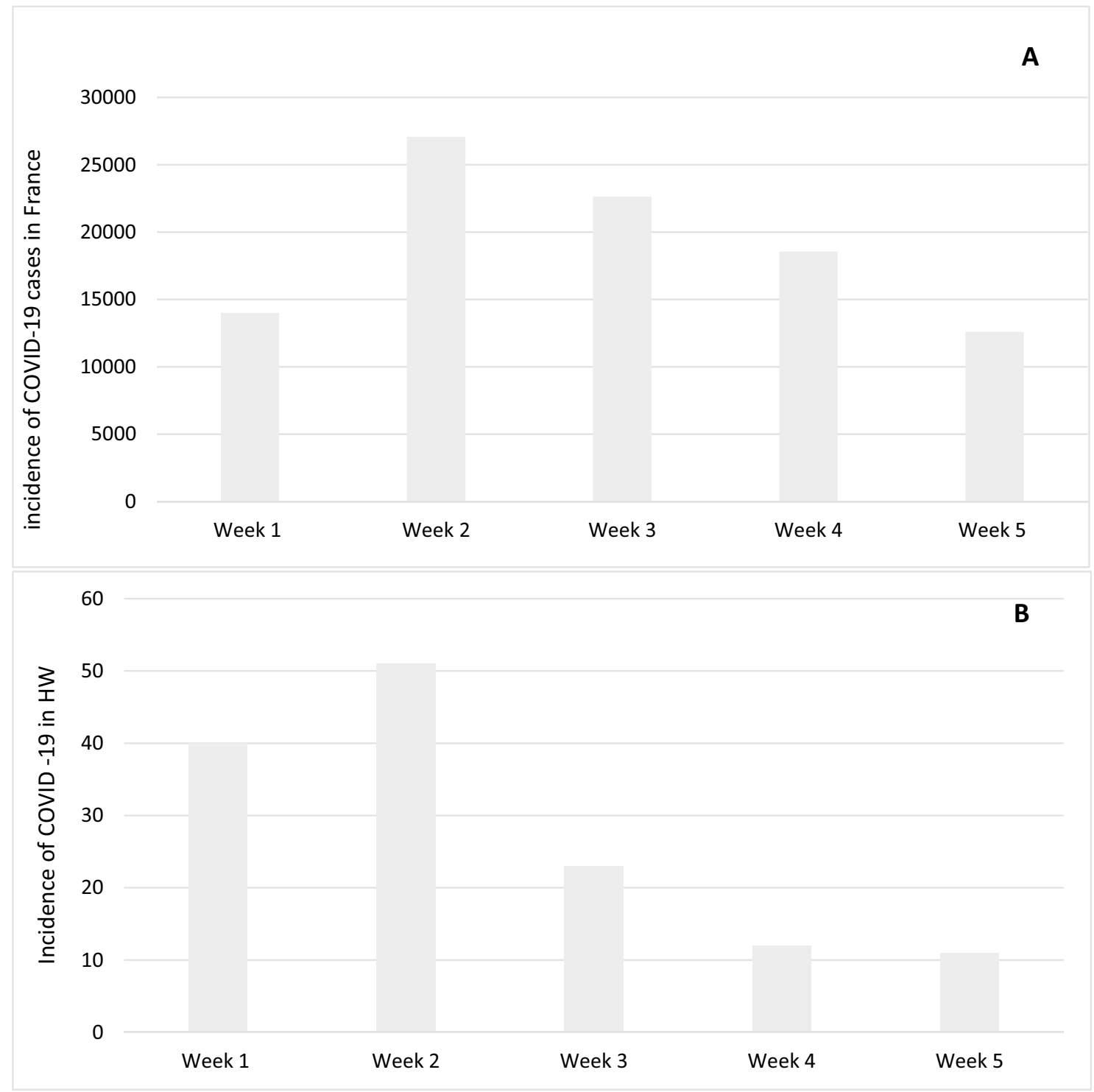

Fig. 2 A Incidence of COVID-19 cases per week in France from 17 March to 20 April. B Incidence of COVID-19 in health workers (HW) from 17 March to 20 April. The period of highest incidence of

not be a sufficient criterion for performing an RT-PCR test, since only $0.3 \%$ of the professionals tested were positive. On the other hand, at the individual level, it can reassure the HWs.

During the study period the number of positive cases did not increase in proportion to the number of samples taken. It is likely that in our study the concern of the HWs, at the height of the pandemic, is at the origin of a resurgence of consultations of asymptomatic HWs because at this period the PPE available was limited.

The distribution of HWs infected by SARS-CoV-2 by age group showed a decrease in the percentage of cases as age increased, especially after the age of 45 . It is possible covid 19 in our study was week 2, from 24 to 30 March 2020, as was the case nationally, before a gradual decrease in the number of new cases in subsequent weeks

that those over 45 years of age, being more experienced and feeling more at risk, may have been better protected or were assigned to less risky services or home confinement. For younger people, they may have felt less concerned or suffered from their lack of experience. The age distribution is consistent with the data collected by two studies (Tostmann et al. 2020; Suárez-García et al. 2020).

Almost half of the positives were nurses or residents/ physicians. Although the percentages could vary significantly from one study to another, the 2 categories of personnel most often contaminated were nurses and resident/ physicians (Tostmann et al. 2020; Keeley et al. 2020; Canova et al. 2020). 


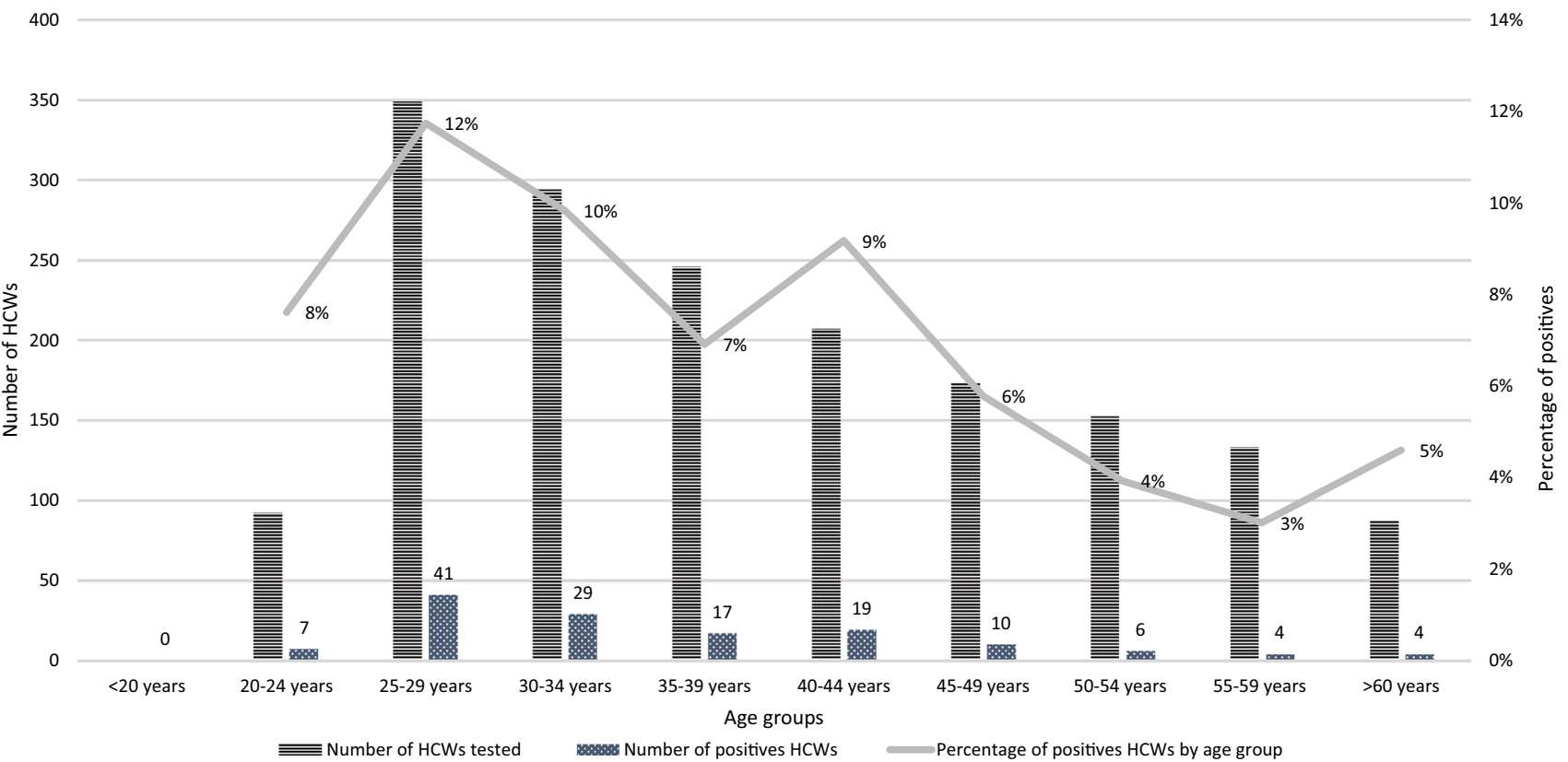

Fig. 3 Distribution of SARS-CoV2 positive healthcare workers (HCWs) by age. The age group 25-29 is the one that has been tested the most and has the most positive tests for SARS COV2 (12\%).

HWs may have been infected by patients (Chang et al. 2020), although transmission of the virus from patients to HWs is not so frequent even without masks (Canova et al. 2020; Cheng et al. 2020; Ghinai et al. 2020; Ng et al. 2020). The risk factors for contamination of HWs by patients were clinical examination and exposure during the administration of nebulization therapy to the patient (Heinzerling et al. 2020). HWs detected as SARS-CoV-2 carriers were mostly working in non-CoVID units; like our study, three studies (Hunter et al. 2020; Porru et al. 2020; Gagneux-Brunon et al. 2020) did not found an increased risk of infection in the most exposed HWs probably because they are more aware of the hazards and risks associated with SARS-CoV-2, wear PPE more strictly, and are better informed and trained. Indeed, a more systematic use of PPE has been shown in the COVID-19 units (Jin et al. 2020). The main protective measures were the wearing of surgical masks by all workers and patients and regular hand washing. In case of contact with a positive or suspected positive patient, the protective equipment to be worn was FFP2 masks, gowns and disposable gloves.

Contamination among HWs also occurred during breaks. This mode of transmission has already been reported (Çelebi et al. 2020) in the context of SARS-COV-2 infection but also for other pathologies with mainly respiratory transmission (Gehanno et al. 1999; Pascual et al. 2006).

In our study no serious cases among HWs were reported, in contrast to the study by Wu et al. which reported $14.8 \%$ serious cases and 5 deaths (Wu and McGoogan 2020). This
There is a decrease in the number of tests performed but also in the rate of positives in the older HW groups

difference may be due to the early exclusion of staff at risk of developing severe forms of COVID-19 or may also be due to early management by a physician specialized in infectious diseases.

\section{Conclusion}

Our study suggests that HWs who tested positive for COVID-19 are often asymptomatic. Therefore, PPE is pivotal to prevent HWs to patients and HWs to HWs transmission during workshifts. Contact tracing and screening is essential to limit the spread of the virus within the hospital. On the other hand, HWs working in COVID-19 units are not more often infected probably because they have a higher risk awareness than other HWs.

Acknowledgements We thank all the secretaries, nurses on the ward and medical students who gave their time.

Author contributions All authors contributed to the study concept, design, and material preparation. The first author performed the data collection. All authors contributed to data analysis. The first draft of the manuscript was written by the first author, and all authors commented on previous versions of the manuscript. All authors read and approved the final version of the manuscript.

Funding No external funding. 
Data availability The data that support the findings of this study are available on request from the corresponding author.

\section{Declarations}

Conflict of interest The authors report no conflict of interest.

Ethical approval Following the opinion of the French Local Ethics Committee for the Protection of Persons (PADS21-39), this study is considered to be outside the scope of the provisions governing biomedical research and routine care.

\section{References}

Barrett ES, Horton DB, Roy J et al (2020) Prevalence of SARS-CoV-2 infection in previously undiagnosed health care workers at the onset of the U.S. COVID-19 epidemic. medRxiv. https://doi.org/10.1101/ 2020.04.20.20072470

Canova V, Lederer Schläpfer H, Piso RJ et al (2020) Transmission risk of SARS-CoV-2 to healthcare workers-observational results of a primary care hospital contact tracing. Swiss Med Wkly 150:w20257. https://doi.org/10.4414/smw.2020.20257

Çelebi G, Pişkin N, Çelik Bekleviç A et al (2020) Specific risk factors for SARS-CoV-2 transmission among health care workers in a university hospital. Am J Infect Control 48:1225-1230. https://doi.org/ 10.1016/j.ajic.2020.07.039

Chang D, Xu H, Rebaza A et al (2020) Protecting health-care workers from subclinical coronavirus infection. Lancet Respir Med 8:e13. https://doi.org/10.1016/S2213-2600(20)30066-7

Cheng VCC, Wong S-C, Chen JHK et al (2020) Escalating infection control response to the rapidly evolving epidemiology of the coronavirus disease 2019 (COVID-19) due to SARS-CoV-2 in Hong Kong. Infect Control Hosp Epidemiol 41:493-498. https://doi.org/ 10.1017/ice.2020.58

Fan J, Liu X, Pan W et al (2020) Epidemiology of coronavirus disease in Gansu Province, China, 2020. Emerg Infect Dis 26:1257-1265. https://doi.org/10.3201/eid2606.200251

Gagneux-Brunon A, Pelissier C, Gagnaire J et al (2020) SARS-CoV-2 infection: advocacy for training and social distancing in healthcare settings. J Hosp Infect 106:610-612. https://doi.org/10.1016/j.jhin. 2020.08.001

Gehanno JF, Pestel-Caron M, Nouvellon M, Caillard JF (1999) Nosocomial pertussis in healthcare workers from a pediatric emergency unit in France. Infect Control Hosp Epidemiol 20:549-552. https:// doi.org/10.1086/501667

Ghinai I, McPherson TD, Hunter JC et al (2020) First known person-toperson transmission of severe acute respiratory syndrome coronavirus 2 (SARS-CoV-2) in the USA. Lancet 395:1137-1144. https:// doi.org/10.1016/S0140-6736(20)30607-3

Greffe S, Espinasse F, Duran C et al (2020) Nasopharyngeal carriage of SARS-CoV-2 among health personnel with symptoms suggestive of COVID-19 in a University Hospital in the Paris suburbs. Rev Med Interne 41:510-516. https://doi.org/10.1016/j.revmed.2020.06.017

Heinzerling A, Stuckey MJ, Scheuer T et al (2020) Transmission of COVID-19 to health care personnel during exposures to a hospitalized patient-Solano County, California, February 2020. MMWR Morb Mortal Wkly Rep 69:472-476. https://doi.org/10.15585/ mmwr.mm6915e5

Hunter E, Price DA, Murphy E et al (2020) First experience of COVID-19 screening of health-care workers in England. Lancet 395:e77-e78. https://doi.org/10.1016/S0140-6736(20)30970-3

Jin Y-H, Huang Q, Wang Y-Y et al (2020) Perceived infection transmission routes, infection control practices, psychosocial changes, and management of COVID-19 infected healthcare workers in a tertiary acute care hospital in Wuhan: a cross-sectional survey. Mil Med Res 7:24. https://doi.org/10.1186/s40779-020-00254-8

Keeley AJ, Evans C, Colton H et al (2020) Roll-out of SARS-CoV-2 testing for healthcare workers at a large NHS Foundation Trust in the United Kingdom, March 2020. Euro Surveill. https://doi.org/10. 2807/1560-7917.ES.2020.25.14.2000433

Krastinova E, Garrait V, Lecam M-T et al (2020) Household transmission and incidence of positive SARS-CoV-2 RT-PCR in symptomatic healthcare workers, clinical course and outcome: a French hospital experience. Occup Environ Med. https://doi.org/10.1136/ oemed-2020-106866

Lagier JC, Colson P, Tissot Dupont H et al (2020) Testing the repatriated for SARS-Cov2: should laboratory-based quarantine replace traditional quarantine? Travel Med Infect Dis 34:101624. https://doi.org/ 10.1016/j.tmaid.2020.101624

Leeds JS, Raviprakash V, Jacques T et al (2020) Risk factors for detection of SARS-CoV-2 in healthcare workers during April 2020 in a UK hospital testing programme. EClinicalMedicine 26:100513. https:// doi.org/10.1016/j.eclinm.2020.100513

Lombardi A, Consonni D, Carugno M et al (2020) Characteristics of 1573 healthcare workers who underwent nasopharyngeal swab testing for SARS-CoV-2 in Milan, Lombardy, Italy. Clin Microbiol Infect 26:1413.e9-1413.e13. https://doi.org/10.1016/j.cmi.2020.06.013

$\mathrm{Ng} \mathrm{K}$, Poon BH, Kiat Puar TH et al (2020) COVID-19 and the risk to health care workers: a case report. Ann Intern Med 172:766-767. https://doi.org/10.7326/L20-0175

Pascual FB, McCall CL, McMurtray A et al (2006) Outbreak of pertussis among healthcare workers in a hospital surgical unit. Infect Control Hosp Epidemiol 27:546-552. https://doi.org/10.1086/506232

Porru S, Carta A, Monaco MGL et al (2020) Health surveillance and response to SARS-CoV-2 Mass testing in health workers of a large Italian hospital in Verona, Veneto. Int J Environ Res Public Health. https://doi.org/10.3390/ijerph17145104

Reusken CB, Buiting A, Bleeker-Rovers C et al (2020) Rapid assessment of regional SARS-CoV-2 community transmission through a convenience sample of healthcare workers, the Netherlands, March 2020. Euro Surveill. https://doi.org/10.2807/1560-7917.ES.2020. 25.12.2000334

Rivett L, Sridhar S, Sparkes D et al (2020) Screening of healthcare workers for SARS-CoV-2 highlights the role of asymptomatic carriage in COVID-19 transmission. Elife. https://doi.org/10.7554/eLife.58728

Suárez-García I, de Aramayona M, López MJ, Sáez Vicente A, Lobo Abascal P (2020) SARS-CoV-2 infection among healthcare workers in a hospital in Madrid, Spain. J Hosp Infect 106:357-363. https:// doi.org/10.1016/j.jhin.2020.07.020

Tostmann A, Bradley J, Bousema T et al (2020) Strong associations and moderate predictive value of early symptoms for SARS-CoV-2 test positivity among healthcare workers, the Netherlands, March 2020. Euro Surveill. https://doi.org/10.2807/1560-7917.ES.2020.25.16. 2000508

Tu Y-F, Chien C-S, Yarmishyn AA et al (2020) A review of SARS-CoV-2 and the ongoing clinical trials. Int J Mol Sci. https://doi.org/10.3390/ ijms 21072657

Wu Z, McGoogan JM (2020) Characteristics of and important lessons from the coronavirus disease 2019 (COVID-19) outbreak in China: summary of a report of 72314 cases from the Chinese center for disease control and prevention. JAMA. https://doi.org/10.1001/jama. 2020.2648

Publisher's Note Springer Nature remains neutral with regard to jurisdictional claims in published maps and institutional affiliations. 